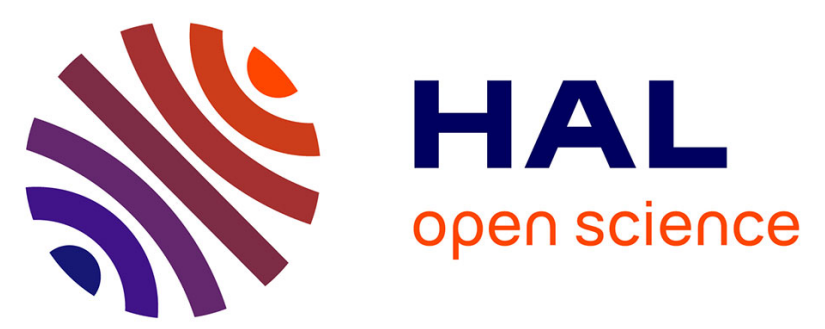

\title{
Analysis of complex mixtures of polyglycerol fatty esters using liquid chromatography and high-resolution mass spectrometry: Retention, structural and relative composition study
}

Jean-Christophe Garrigues, Marion Cournac, Marlène Oswald, Nicolas Ritter,

Muriel Blanzat, Stéphanie Cassel

\section{To cite this version:}

Jean-Christophe Garrigues, Marion Cournac, Marlène Oswald, Nicolas Ritter, Muriel Blanzat, et al. Analysis of complex mixtures of polyglycerol fatty esters using liquid chromatography and highresolution mass spectrometry: Retention, structural and relative composition study. Journal of Chromatography A, 2020, 1616, pp.460792. 10.1016/j.chroma.2019.460792 . hal-02614161

\author{
HAL Id: hal-02614161 \\ https://hal.science/hal-02614161
}

Submitted on 23 Nov 2020

HAL is a multi-disciplinary open access archive for the deposit and dissemination of scientific research documents, whether they are published or not. The documents may come from teaching and research institutions in France or abroad, or from public or private research centers.
L'archive ouverte pluridisciplinaire HAL, est destinée au dépôt et à la diffusion de documents scientifiques de niveau recherche, publiés ou non, émanant des établissements d'enseignement et de recherche français ou étrangers, des laboratoires publics ou privés. 
Analysis of complex mixtures of polyglycerol fatty esters using liquid chromatography and high-resolution mass spectrometry: retention, structural and relative composition study.

Jean-Christophe Garrigues ${ }^{\mathrm{a}, *}$, Marion Cournac ${ }^{\mathrm{a}}$, Marlène Oswald ${ }^{\mathrm{a}}$, Nicolas Ritter ${ }^{\mathrm{b}}$, Muriel Blanzat $^{\mathrm{a}}$, Stéphanie Cassel $^{\mathrm{a}, *}$

(1)

a Laboratoire des IMRCP, Université de Toulouse, CNRS UMR 5623, Université Paul Sabatier, 118 route de Narbonne, 31062 Toulouse Cedex 9, France

${ }^{\mathrm{b}}$ Gattefossé SAS, 36 Chemin de Genas, 69800 Saint Priest, France

* Corresponding authors. E-mail address: cassel@chimie.ups-tlse.fr (Stéphanie Cassel), garrigues@ chimie.ups-tlse.fr (Jean-Christophe Garrigues)

(1)

\section{Abstract}

Polyglycerol esters (PGEs), produced by esterification of fatty acids on polyglycerols, were analysed by High Resolution Mass Spectrometry (HRMS), HPLC-MS and U-HPLC-MS. A structural study of PGEs in 4 samples synthesised by the Gattefossé company was carried out using an elemental analysis of HRMS spectra and modelling of all probable isomers and cyclic structures. The results were used to construct a structural database of all species present in the 4 samples. After an assessment of the selectivity of 5 reversed phase columns: Aeris Widepore

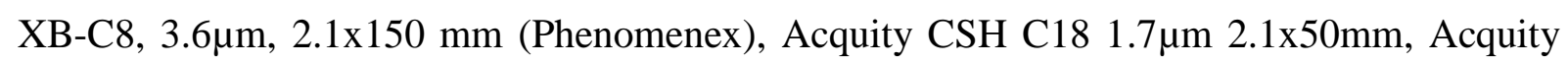

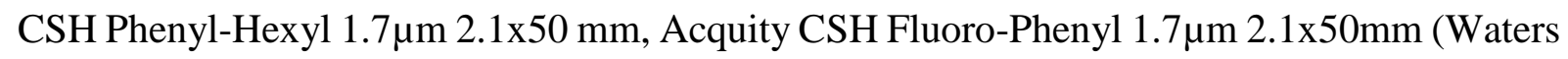
Co.) and Kinetex F5 1.7 $\mu \mathrm{m}$ 2.1x100mm (Phenomenex), HPLC-MS and U-HPLC-MS analyses were performed on an Aeris Widepore XB-C8 (Phenomenex) column (HPLC) and Acquity CSH Fluoro-Phenyl (Waters) column (U-HPLC) with aqueous formic acid /acetonitrile in 
gradient mode. The separation was optimised with 10 minutes (HPLC) and 5 minutes (UHPLC) of gradient. The detection, performed on a QDA detector (Waters), produced extracted ion chromatograms (XICs) based on all adducts identified in the HRMS analysis. HPLC and U-HPLC analyses showed the different mono- and di-ester species and provided relative quantification of all identified constituents. The combined analyses of the HRMS, HPLC-MS and U-HPLC-MS results were used to compare the different PGE batches and quantify the molecular constituents according to their relative abundance, for these complex mixtures. With HPLC and U-HPLC analyses, using 2 different gradient times and 2 different selectivity columns, and comparing the retention factors and $\log \mathrm{P}$ of the different species, it was possible to link structural identification and relative quantification of all PGEs identified in the samples.

\section{Highlights}

- Batch comparisons and quantification of constituents of complex formulations

- Structural database construction with HRMS spectra and modelling of all probable PGEs

- HRMS and (U)-HPLC-MS can link structural identification to relative PGEs quantification

\section{Keywords}

HPLC; HRMS; Formulation; Complex matrices; Polyglycerol esters; Green surfactant.

\section{Introduction}

Polyglycerol is a viscous, non-toxic, water-soluble polyether with a high hydrophilic character and excellent biocompatibility. Polyglycerol esters (PGEs), obtained via esterification of fatty 
acids on polyglycerols, are odourless, biodegradable non-ionic surfactants. They are mainly used in cosmetics, the food industry and pharmaceuticals and they come from renewable resources [1-3]. These products have excellent skin tolerance, better thermal stability than other conventional non-ionic surfactants, and moderate antimicrobial activity. Furthermore in addition to their surface-active properties, the lipophilic part, consisting of a fatty acid, combined with a hydrophilic part based on glycerol, gives them emollient and moisturizing properties [4]. PGEs are considered as green surfactants with promising physicochemical properties.

Polyglycerol can be synthesised from glycidol [5] but for safety concern glycerol is preferred. Glycerol is a safe, renewable material generated as a by-product during the biodiesel process [6]. The polymerisation of glycerol is ideally conducted at a high temperature in the presence of metal hydroxide $[7,8]$ or more selective catalysts $[9,10]$. As previously mentioned, polyglycerol esters are produced from the esterification of fatty acids, derived from triglycerides of vegetable oils, and polyglycerols. Accordingly, polyglycerol esters are made of a complex mixture of compounds depending on the degree of glycerol polymerisation, on the polyglycerol branching isomers and on the number and type of fatty acids that have reacted per molecule of surfactant. It is important to determine the composition of these complex mixtures both qualitatively and quantitatively. Indeed, the properties of PGEs are connected to their composition [11]. Various chromatographic techniques are available for analysing PGEs: thinlayer [12], GC [13], RP-HPLC [14] and SFC [15]. Specific methods have also been developed by combining GC and HPLC analyses [16] or using stationary phases of porous graphitic carbon stationary phases [17]. In this study, the complexity of 4 mixtures of PGEs was analysed by direct infusion in a Q-ToF HRMS system. With these structural data, PGEs retention were studied on five stationary phases (one HPLC and four U-HPLC) in reversed mode, in order to optimise methods adapted to the total range of PGE structures. By integrating molecular 
modelling data, we connected the PGEs' retention factors to specific mono-, di- and tri-esters. These identified structures were then quantified in relative quantity in the 4 mixtures.

\section{Materials and methods}

\subsection{Synthesis}

\subsubsection{Chemicals}

Products used for synthesis: polyglycerol (Spiga spa, Genova, Italy), stearic acid 98\% (Mosselman) and technical oleic acid (Berg \& Schmidt).

\subsubsection{Procedure for PG distillation}

Prior to the synthesis, diglycerol was removed by means of short path molecular distillation (UIC GmbH) to increase the content in polyglycerols with a higher degree of polymerisation. The short path distillation unit (UIC GmbH) was equipped with temperature regulators (Julabo ME4, HT30M1) and connected to a cold trap filled with liquid nitrogen. The distillation conditions were the following: feeding the polyglycerol- 6 into the distillation unit at $80^{\circ} \mathrm{C}$ and $300 \mathrm{~mL} / \mathrm{h}$, pressure $0.05 \mathrm{mbar}$, heating mantle set at $200^{\circ} \mathrm{C}$ and internal condenser at $90^{\circ} \mathrm{C}$.

\subsection{Analytical methods}

\subsubsection{Chemicals}

HPLC-grade acetonitrile $(\mathrm{ACN})$, methanol $(\mathrm{MeOH})$ and formic acid $(\mathrm{HCOOH})$ were obtained from Sigma-Aldrich Co. (St. Quentin Fallavier, France). Water $\left(\mathrm{H}_{2} \mathrm{O}\right)$ was purified using a milli-Q purification system (Millipore, St. Quentin Yvelines, France).

\subsubsection{HRMS}

Solutions (1 mg.mL $\left.\mathrm{m}^{-1}\right)$ of samples $\mathrm{S} 1, \mathrm{~S} 2, \mathrm{~S} 3$ and $\mathrm{S} 4$ were obtained by dissolution in $\mathrm{MeOH}$ with $0.1 \% \mathrm{HCOOH}$, sonication at $20^{\circ} \mathrm{C}$ and filtration through $0.2 \mu \mathrm{m}$ nylon filters (Waters 
Corporation, Milford, MA, USA). The samples were injected $(5 \mu \mathrm{L})$ without using a column on a Xevo G 2 QTof (Waters) system, with elution water 0.1\% HCOOH/ACN (90\%:10\% v:v) and flow $0.5 \mathrm{~mL} \cdot \mathrm{min}^{-1}$, capillary voltage ESI+ $(3 \mathrm{kV})$, fragmentor potential $(30 \mathrm{~V})$, nitrogen ESI $\left(800 \mathrm{~L} \cdot \mathrm{H}^{-1}\right)$, nitrogen cone $\left(20 \mathrm{~L} \cdot \mathrm{H}^{-1}\right)$ and source temperature $\left(110^{\circ} \mathrm{C}\right)$. Masslynx was used to process the results. After summation of 1 min of acquisition, all spectra were analysed and compounds identified by comparison with the theoretical mass with a maximum deviation of 3 ppm.

The deviation is calculated as shown below:

$\Delta \mathrm{ppm}=(\mid$ theoretical mass - experimental mass $\mid) /$ theoretical mass $\times 1000000$

\subsubsection{HPLC-MS and U-HPLC-MS}

HPLC and U-HPLC analyses were performed on an Acquity ${ }^{\circledR}$ UPLC H-Class system composed of a quaternary solvent manager, a sample FTN manager and an Acquity ${ }^{\circledR}$ QDA detector (Waters Corporation, Milford, MA, USA). Analyses were monitored by Empower 3 software (Waters Co.). Eluents were filtered through $47 \mathrm{~mm}, 0.45 \mu \mathrm{m}$ nylon membrane discs (Waters). HPLC separations were carried out on Aeris Widepore XB-C8, 3.6 $\mu \mathrm{m}, 2.1 \times 150 \mathrm{~mm}$ (Phenomenex, Torrance, CA, USA) and U-HPLC separations were carried out on 4 columns:

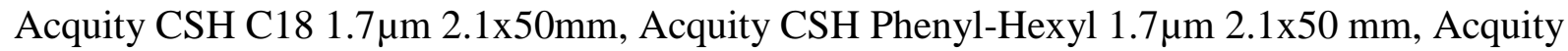

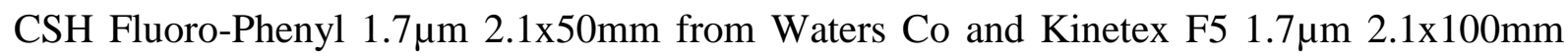
(Phenomenex).

For HPLC analysis, a gradient mode was used with all time conditions : (\% vol.) t 0 min: $40 \%$ $\mathrm{H}_{2} 0,50 \%$ ACN, $10 \% \mathrm{ACN}+1 \% \mathrm{HCOOH}$; $\mathrm{t}_{10 \mathrm{~min}}: 90 \% \mathrm{ACN}, 10 \% \mathrm{ACN}+1 \% \mathrm{HCOOH}$; Flow $1 \mathrm{~mL} \cdot \mathrm{min}^{-1}$; the run time was 10 minutes and the column temperature was $25^{\circ} \mathrm{C}$. For U-HPLC analysis, all-time conditions were (\% vol.) $\mathrm{t}_{0 \min }: 50 \% \mathrm{H}_{2} 0,40 \% \mathrm{ACN}, 10 \% \mathrm{ACN}+1 \%$ $\mathrm{HCOOH}$; $\mathrm{t}_{5 \min }: 90 \% \mathrm{ACN}, 10 \% \mathrm{ACN}+1 \% \mathrm{HCOOH}$; Flow $0.5 \mathrm{~mL} \cdot \mathrm{min}^{-1}$; the run time was 5 
minutes and the column temperature was $35^{\circ} \mathrm{C}$. MS analyses were performed with an electrospray ionisation source (ESI) in positive mode with a sampling frequency of $8.8 \mathrm{~Hz}$. The capillary and fragmentor potentials were set respectively to $0.8 \mathrm{kV}$ and $15 \mathrm{~V}$. The QDA analyser worked in full scan mode, and the mass range was set at $m / z$ 145-1250. To identify and analyse each PGE, full-scan survey spectra were used to generate extracted ion chromatograms (XICs) using the $[\mathrm{M}+\mathrm{Na}]^{+}$or $[\mathrm{M}+\mathrm{H}]^{+}$mass value adjusted at 2 decimals, from theoretical or experimental masses (Table $\mathrm{S} 1$ to $\mathrm{S} 4$ in supplementary data). For the semi-quantitative analysis of each PGE, the peaks obtained for each XIC were integrated and the relative abundance was calculated by the ratio of each PGE's peak area on the sum of the integrated peaks areas.

\subsection{Molecular modelling}

All PGE structures were optimised using semi-empirical AM1 quantum mechanical calculations in the MOPAC application, using Chem 3D Pro version 14.0 software (CambridgeSoft) with an RMS gradient of 0.100 to minimise energy. The optimised structures were used to calculate the octanol/water partition coefficient (log P) and Connolly molecular area (CMA) with Chem 3D Pro 14.0 software (CambridgeSoft).

\section{Results and discussion}

\subsection{Modelling of structures}

Prior to the experiments, modelling was performed for all the structures that could possibly be present. This comprised free polyglycerols (PG), with a degree ranging from 1 to 12, and including possible loss of water molecules, whether because of intrinsic cyclic structures or dehydration processes in the mass spectrometer. Then the same kind of work was done for fatty esters, combining every single free PG with up to 12 fatty chains. Since the analysis of PGEs 
mixtures by HRMS, HPLC and U-HPLC-MS did not resolve all probable isomers and branched PGs, modelling was limited to linear PGs with 1, 2 or 3 chains of esterified fatty acids in terminal positions for mono and di-esters. For tri-esters models, the $3^{\text {rd }}$ fatty chain is positioned on the central OH function of the PGs. For PGs and PGEs with water molecule loss, the models incorporate 1 or 2 water loss.

The molecules will be named as follows:

- $\quad$ PGn $=$ free polyglycerol with a polymerisation degree $n$

- $\quad P G[x]=P G$ with a loss of $x$ water molecules (maximum 2)

- $\quad$ PGn+mCx $=$ PGE with PGn and m linear chains of $x$ carbon atoms, for a set of four possible fatty acids (C16, C18, C18:1 and $\mathrm{C} 18: 2)$

- $\quad \mathrm{PGn}+\mathrm{mCx} / \mathrm{pCy}=\mathrm{PGE}$ with PGn and $\mathrm{m}$ linear chains of $\mathrm{x}$ carbon atoms and $\mathrm{p}$ linear chains of y carbon atoms, for a set of four possible fatty acids (C16, C18, C18:1 and C18:2)

- $\quad[\mathrm{M}+\mathrm{H}]^{+}=$ionic structure of a PGE with a hydrogen adduct

- $\quad[\mathrm{M}+\mathrm{Na}]^{+}=$ionic structure of a PGE with a sodium adduct

For the study, we had samples of the mixtures of the free PGs used in the esterification reaction, one centred around PG3 and two around PG6 (either distilled or not).

We had 4 samples of PGEs obtained with different fatty acid mixtures, whose initial reactive compositions are given in Table 1 below.

\begin{tabular}{ccc}
\hline Sample & Free PGs & Fatty acids \\
\hline S1 & Distilled PG6 & C18 (98.8\%) \\
S2 & Distilled PG6 & C18:1 (79.4\%)
\end{tabular}


C18:2 (12.4\%)

C16 (4.8\%)

C18 (1.9\%)

S3

Non distilled PG6

C18 (50\%)

C16 (50\%)

S4

PG3

C18:1 (79.4\%)

C18:2 (12.4\%)

C16 (4.8\%)

C18 (1.9\%)

Table 1. Samples S1 to S4 initial reactive composition.

Then, for every possible molecule, the molecular formula was determined, and the exact mass at the fourth decimal level was calculated for both hydrogen and sodium adducts (which may form during the synthesis process or in the ESI source).

Possible molecules are modelled and three-dimensional structures minimised, for calculation of the physicochemical parameters $\log \mathrm{P}$ and Connolly molecular area. These physicochemical parameters will provide information in the structural study on possible formulae, linking the HRMS and experimental retention data obtained using the two chromatographic methods.

\subsection{HRMS analyses}

Once this preliminary work was done, all 4 samples were analysed using high resolution mass spectrometry in order to identify all the molecules present in each sample. Figure 1 shows the ESI+ TOF mass spectrum obtained with sample S3. In all acidified and dissolved methanol samples, $[\mathrm{M}+\mathrm{H}]^{+}$and $[\mathrm{M}+\mathrm{Na}]^{+}$adducts were detected in ESI positive mode. This can be explained by the ability of the polyglycerol polar-head group to behave as a relatively good chelators for sodium ions, depending on the PG structure. The analysis of these complex HRMS 
spectra consisted of elemental analysis of the PGEs corresponding to the molecular formulae of the modelled PGEs for signals greater than $10 \%$ of the relative intensity.

For S1, (Table S1, supplementary data) obtained from distilled PG6 and C18 fatty acid, esters with a polar head comprising between 3 and 8 glycerol units combined with one or two fatty chains were mainly detected, as expected as this is consistent with the starting materials used. In this sample, 27 PGEs were found with a relative intensity above $10 \%$ for 143 modelled, corresponding to $19 \%$ identification score.

For S2, (Table S2, supplementary data) which resulted from the reaction between distilled PG6 and a mixture of fatty acids mainly composed of oleic acid, here again esters with a polar head comprising between 3 and 8 glycerol units esterified by one to up to 3 fatty chains were detected. For compounds that have two or more chains, one cannot distinguish between a combination of $2 \mathrm{C} 18: 1$ and $1 \mathrm{C} 18+1 \mathrm{C} 18: 2$, for they have the exact same formulas and therefore theoretical mass. Nonetheless, as there is nearly $80 \%$ of oleic acid and only less than $2 \%$ stearic acid in the starting mixture of fatty acids used to prepare $\mathrm{S} 2$, it is statistically most likely that it is the combination of $2 \mathrm{C} 18: 1$ fatty chains. In this sample, 33 PGEs were found with a relative intensity above $10 \%$ for 327 modelled, corresponding to $10 \%$ identification score.

S3 (Table S3, supplementary data was obtained from the reaction between non distilled PG6 and a 1:1 mixture of palmitic and stearic acid. As expected, many more compounds can be detected here as diglycerol and other fairly volatile compounds (cyclic ones) were not removed by the distillation step, as in S1 and S2. PG2 to PG7 combined with up to 3 chains can therefore be detected in S3. In sample S3, 59 PGEs were found with a relative intensity above $10 \%$ for 150 modelled, corresponding to $39 \%$ identification score. 
213 Finally, S4 (Table S4, supplementary data was obtained by the combination of PG3 with the 214 same fatty acid mixture as for S2, mainly composed of oleic acid. As expected, PG2 to PG5 215 bearing up to 3 chains can be found in the mixture, with the same uncertainty for compounds 216 having 2 or more chains, whether 2 C18:1 or $1 \mathrm{C} 18+1 \mathrm{C} 18: 2$. Same conclusion applies here. . 217 In sample S4, 32 PGEs were found with a relative intensity above $10 \%$ for 327 modelled, 218 corresponding to $10 \%$ identification score.

In every sample mixture, non-reacted free PGs can be detected, depending on the starting free PGs mixture, along with the esters.

222 In all mixtures, no PG with a degree exceeding 12 was found, even at very low level.

223 No structure with more than 2 water loss was detected.

Table 2 summarises the rough composition of each sample.

\begin{tabular}{llll}
\hline Sample & Free PGs & PG esters \\
S1 & PG3 to PG8 & PG3 to PG8 with up to 2 C18 chains \\
S2 & PG3 to PG6 & PG3 to PG7 with up to 3 chains / mixed grafting \\
& & (C16/C18/C18:1/C18:2) \\
S3 & PG2 to PG8 & PG2 to PG7 with up to 3 chains / mixed grafting (C16/C18) \\
S4 & PG3 to PG4 & PG3 to PG5 with up to 3 chains / mixed grafting \\
& & (C16/C18/C18:1/C18:2)
\end{tabular}

\subsection{HPLC-MS}

229 In order to check that the analytical methods ensure the separation and identification of the 230 various classes of PGEs, we chose test compounds, for which the probable developed formulas 
are given in Figure 2 and optimised structures after AM1 minimisation are given in Figure S1 (supplementary data). The esterification reaction involves the terminal hydroxyl of each PG, as they are more reactive than the other $\mathrm{OH}$ groups [18]. The terminal positions are therefore chosen in all models to represent the monoesters and di-esters. The test compounds were selected to simplify method development and to verify that they could be separated on the columns used in this study.

These 4 test compounds were identified by HRMS in sample S3 (table S4, supplementary data). We chose esterified PGEs from PG4, 2 monoesters with a single C16 and a single C18 chain and 2 di-esters with $2 \mathrm{C} 16$ and 2C18 chains. Because the exact structures and positions of the fatty acids are unknown, the formulae given in Figure 2 are only probable structures, in reference to the more reactive terminal hydroxyl groups. We used these four test compounds because they show strong variations in their hydrophobic $(\log \mathrm{P})$ and steric (Connolly molecular area) values (Table 3) and enable us to assess the selectivity of the stationary phase and the conditions of gradient on these model PGEs with varied steric and hydrophobicity footprints. These hydrophobic and steric parameters are used for modelling the reversed-phase retention mechanisms [19] and are therefore relevant indicators of the structural variability of interactions.

\begin{tabular}{cccc}
\hline Compound & $\begin{array}{c}\text { Compound } \\
\text { code }\end{array}$ & $\log \mathrm{P}$ & Connolly Molecular Area $\left(\AA^{2}\right)$ \\
\hline PG4[1]+1C16 & A & 4.8 & 617.9 \\
PG4[1]+1C18 & B & 5.9 & 654.9 \\
PG4+2C16 & C & 12.1 & 932.7 \\
PG4[1]+2C18 & D & 15.2 & 993.0 \\
\hline
\end{tabular}


Table 3. $\log \mathrm{P}$ and Connolly molecular area calculated for PG4[1]+1C16, PG4[1]+1C18, PG4+2C16, PG4[1]+2C18.

The XIC chromatogram of sample S3 (Figure 3) shows the separation of the 4 PGEs from the least hydrophobic (A: $\log \mathrm{P} 4.8$ ) to the most hydrophobic (D: $\log \mathrm{P} 15.2)$ in less than 10 minutes, with a simple aqueous gradient using $\mathrm{ACN}$ as an organic modifier. This XIC chromatogram was obtained by summation of $\mathrm{m} / \mathrm{z}$ : 557.36, 563.41, 813.60 and 851.65 . Each separated chromatograms obtained with the $4 \mathrm{~m} / \mathrm{z}$ values is given in Figure S2 (supplementary data).

Wide-pore core-shell columns showed good efficiency and peak capacity for the separation of macromolecules [20]. The Aeris ${ }^{\mathrm{TM}}$ WIDEPORE XB-C8 $3.6 \mu \mathrm{m}$ silica particles, surrounded by a $0.2 \mu \mathrm{m}$ porous layer, showed good selectivity in the separation of the 4 test PGEs. The semiquantitative analysis was performed from XIC chromatograms for all PGEs theoretically present in each sample. Tables 4 to 6 present the results for samples S1 to S4 for relative abundances above $1 \%$.

For S1 (Table S5, supplementary data), the major species were PG4, PG5 and PG6 esters containing two $\mathrm{C} 18$ chains, accounting for 7.3 to $8.1 \%$ (relative abundance) of the mixture. There was also $5.9 \%$ PG3+2 C18. Di-esters accounted for $35.7 \%$ of the S1 sample. The majority-identified monoesters were PG3 and PG4 with one C18 chain, at 6.5 and $4.6 \%$ respectively. Other classes of monoesters were found with different PGs with loss of a water molecule: PG3[1], PG4[1], PG5[1] and PG6[1] associated with one C18 chain. Monoesters accounted for $42.5 \%$ of the S1 sample. The remaining $21.8 \%$ were made up of 70 free PGs, mono-and di-ester minority species, all identified with this analytical method. All free PGs were found at retention times between 0.20 and 0.35 minutes. Monoesters are found between 0.59 and 1.96 min and di-esters separated between 7.3 and 9.3 min. PG8, PG6, PG5 and PG3 esters with one C18 chain are found with a loss of one water molecule with higher retention times. 
For PG8[1], PG6[1], PG5[1] and PG3[1] esters with one C18 chain, this water loss occurred prior to separation during the esterification reaction. For PG7[1], PG4[1], and PG3[1] ] esters with one C18 chain, This water loss can also occur in the ESI source and the water-loss PGEs are then identified at the same retention time as linear PGEs.

For S2 (Table S6, supplementary data), a very complex mixture obtained from distilled PG6, the analysis showed that the major species were PG3, PG4, PG5 and PG6 esters bearing two alkyl chains (C18, C16, C18:1, C18:2), accounting for 13.5 to $6.9 \%$ (relative abundance) of the mixture. The simple quadrupole system resolution does not discriminate isobaric compounds. The PGE identified with an experimental mass of 791.78 can be attributed to either PG3+1C18+1C18:2, PG3+2C18:1 or PG4+2C16. As the C18:1 fatty acid accounts for nearly $80 \%$ of the fatty acid mixture used for the preparation of sample 2 , we can only suggest that PG3+2C18:1 may be the major compound having this mass. Di-esters account for $50.1 \%$ of sample S2. The majority-identified monoesters were PG3, PG4 and PG4[1] with one C18:1 chain, at 4.6, 4.1 and 3.0\% respectively. Monoesters account for $26.9 \%$ of the S2 sample. The remaining $23 \%$ made up of 70 free PGs, mono and di-ester minority species. Most of the monoesters and di-esters with one or two identified water loss are found at the same retention time as linear PGEs, this mechanism is linked to the ESI source.

For S3 (Table S7, supplementary data), a complex mixture of non-distilled PG6 with C18 and C16, the analysis showed that the major species were PG4 esters containing one C18 and one C16 alkyl chains and PG3[1] monoester with one C18 chain, accounting for 6.6 and $6.1 \%$ (relative abundance) of the mixture, respectively. Di-esters account for $46.9 \%$ of the $\mathrm{S} 3$ sample. The majority-identified monoesters comprised all the PGs from PG2 to PG8[1] with one C18 or one C16 chain. Monoesters accounted for $25.7 \%$ of sample S3. The remaining $27.4 \%$ were made up of 65 free PGs, mono and di-ester minority species. PG3, PG4, and PG5 esters with 
one $\mathrm{C} 16$ or one $\mathrm{C} 18$ chain are found with a loss of one water molecule with higher retention times.This water loss occurred prior to separation during the esterification reaction.

The S4 sample analysis (Table S8, supplementary data) showed results close to S2. The major species were PG3, PG4 and PG5 di-esters containing C18, C18:1 and C18:2 alkyl chains, accounting for 19.3 and $11.5 \%$ (relative abundance) of the mixture. Di-esters account for $55.9 \%$ of the S4 sample. The majority-identified monoesters comprised PG3 and PG4 with one C18:1 chain. Monoesters accounted for $21.2 \%$ of the S4 sample. The remaining $27.4 \%$ were made up of 60 free PGs, mono and di-ester minority species. As observed for sample S2, most of the monoesters and di-esters with one or two identified water loss are found in S4 at the same retention time as linear PGEs, this mechanism is linked to the ESI source.

\subsection{U-HPLC-MS analyses}

The first work was to select the suitable column for separating the PGEs according to the chosen gradient. We followed the 4 typical PGEs (Figure 2), by injecting sample S3 for the 4 study columns with a XIC chromatogram extraction for each compound. With the Acquity CSH C18 column, only PG4[1]+1C16 and PG4[1]+1C18 were identified. With the CSH Phenyl-Hexyl and Kinetex F5 columns, the 2 test monoesters PG4[1]+1C16, PG4[1]+1C18 and one di-ester PG4+2C16 were identified.

The XIC chromatogram of sample S3 (Figure 4) shows the separation of the 4 test PGEs on the Acquity fluoro-phenyl column in less than 5 minutes, with a simple aqueous gradient using ACN as an organic modifier. The column selectivity of the Acquity fluoro-phenyl column was adapted to the separation of the test PGEs in a wide range of log Ps (from 4.8 to 15.2) and Connoly molecular areas (from 617 to 993), (Table 2). The Acquity fluoro-phenyl column was 
selected for all identification and semi-quantitative analysis of the PGEs samples, with the UHPLC method. These analytical conditions gave an equivalent resolution compared to the HPLC method (Figure 3) while dividing the analysis time by 2. This XIC chromatogram was obtained by summation of $\mathrm{m} / \mathrm{z}$ : 557.36, 563.41, 813.60 and 851.65. Each separated chromatograms obtained with the $4 \mathrm{~m} / \mathrm{z}$ values is given in Figure S3 (supplementary data).

The semi-quantitative analysis was performed from XIC chromatograms for all PGEs theoretically present in each sample. Tables S9 to S12 summarise the results for samples $\mathrm{S} 1$ to S4 for relative abundances above $1 \%$. For sample S1 (Table S9, supplementary data), the UHPLC method provided identification of 37 compounds with more than $1 \%$ relative abundance, which is 24 additional compounds compared to the HPLC method. It is possible with the UHPLC to identify 6 free PGs (PG3 to PG7) at an rt close to $0.31 \mathrm{~min}$, accounting for between 1.0 and $1.5 \%$ of all PGEs and PGs detected. The major species were PG3 and PG4 esters containing two C18 chains, accounting for 6.1 and $5.81 \%$ (relative abundance) of the mixture. All the most abundant di-esters identified by HPLC, from PG3 to PG6, detected and quantified with similar yields. With U-HPLC, di-esters accounted for 36\% (35.7\% with HPLC), monoesters accounted for $37.1 \%$ (42.5\% with HPLC) and free PGs were quantified at $6.9 \%$ (ND with HPLC) of the S1 sample. The remaining $20 \%$ are made up of free PGs, mono and di-ester minority species. For sample S2 (Table S10, supplementary data), the U-HPLC method provided identification of 33 compounds with more than $1 \%$ of relative abundance, which is 7 additional compounds compared to the HPLC method. With the U-HPLC analysis, the majority identified species were PG3, PG4, PG5 and PG6 esters containing two alkyl chains (C18, C16, C18:1, C18:2), accounting for between 7.9 and 3.3\% (relative abundance) of the mixture. All the most abundant di-esters identified by HPLC, from PG3 to PG6, were detected and quantified with similar yields except for the compound with an experimental mass of 791.71 attributed to 
$[\mathrm{PG} 3+1 \mathrm{C} 18+1 \mathrm{C} 18: 2+\mathrm{Na}]^{+}$or $[\mathrm{PG} 3+2 \mathrm{C} 18: 1+\mathrm{Na}]^{+}$or $[\mathrm{PG} 4+2 \mathrm{C} 16+\mathrm{H}]^{+}$identified at a relative abundance of $7.9 \%$ with the U-HPLC method and $13.5 \%$ with the HPLC method. Di-esters represent $35.8 \%$ (50.1\% with HPLC), and monoesters $26.9 \%$ (25.6\% with HPLC). For S3

351

(Table S11, supplementary data), a complex mixture of non-distilled PG6 with C18 and C16, the analysis showed that the major species were PG3[1] monoester containing one C18 alkyl chains and the PG4[1] di-ester with one $\mathrm{C} 18$ and one $\mathrm{C} 16$ chain, accounting for $8 \%(6.1 \%$ with HPLC) and $2.9 \%$ (6.6\% with HPLC) of the mixture, respectively. Di-esters accounted for $33.9 \%$ and monoesters for $29.5 \%$ of sample S3. One free PG was also identified at $1.1 \%$ (PG4[1]). The S4 analysis (Table S12, supplementary data)showed results close to S2 and to the HPLC analysis. The major species were PG3, PG4 and PG5 di-esters containing C16, C18, C18:1 and C18:2 alkyl chains, accounting for 10.1\% (19.3\% with HPLC) 5.6\% (11.5\% with HPLC) and 5.2\% (5.6\% with HPLC) of the mixture. Di-esters accounted for $40.4 \%$ of the S4 sample. The majority-identified monoesters comprised PG3 and PG2 and PG4 with one C18:1 chain. Monoesters accounted for $28.8 \%$ of sample S4.

Isobaric ions can be detected with both HPLC and U-HPLC methods. The adduct $\mathrm{m} / \mathrm{z} 749.80$ can be detected in sample S2, S3 and S4 with the U-HPLC-MS method. This signal can be attributed to 2 different PGEs: PG3[1]+1C18+1C16, with a calculated $\log$ P of 20.9 and a Connolly molecular area $876.3 \AA^{2}$ or PG6+1C18:1, with a calculated log P of 2.8 and a Connolly molecular area $811.7 \AA^{2}$. For samples S2 and S4, the analysis of the low retention time of the corresponding PGE (0.7 min) was compatible with PG6+1C18:1, with lower values for the calculated log P and Connolly molecular area (Table S10 and Table S12, supplementary data). For sample S3, the retention time of this PGE was higher (3.4 min) and compatible with a di-ester, PG3[1]+1C18+1C16, with a high value for $\log \mathrm{P}$ and the Connolly molecular area (Table S11, supplementary data). The analysis done with the HPLC-MS method detected m/z 749.68 in sample S2 and S4, with low retention time values(0.6 min, Table S6 and Table S8), 
compatible with the monoester PG6+1C18:1, as analysed with the U-HPLC-MS method. This signal was not detected in the S3 sample. The comparison of the retention time and the molecular calculated parameters log P and Connolly molecular area, can be used to discriminate probable PGEs structure for isobaric ions.

HRMS results confirm the interpretation given with the comparison of the retention time with the calculated values for $\log \mathrm{P}$ and the Connolly molecular area. In sample S2, the elemental analysis confirmed the probable formulae of PG6+1C18:1 (Table S2, supplementary data), and for S3 the identification of the di-ester PG3[1]+1C18+1C16 (Table S3, supplementary data).

The development of the two HPLC-MS and U-HPLC-MS methods is complementary in analysing these complex matrices. Some di-esters of the S3 sample, such as PG3[1]+1C18+1C16, are identified by the U-HPLC MS method (m/z: 749.80, Table S11, supplementary data) and not detected by the HPLC-MS method. These very hydrophobic diesters (log P: 20.9) have a high steric effect with the stationary phase (Connolly molecular area $876.3 \AA^{2}$ ) and are therefore not correctly eluted by the HPLC-MS method, whereas this PGE is identified with HRMS (Table S3, m/z: 749.5917, supplementary data). The simple HRMS analysis of the samples, without chromatographic separation, cannot identify some of the less apolar monoesters, such as PG6+1C18:1. This PGE was identified well in sample S2 using HRMS (Table S2, m/z: 749.4655) and was also identified with the HPLC-MS and UHPLC-MS methods, with a relative abundance ranging from $1.3 \%$ to $1.4 \%$ (Table S6 and S10, m/z 749.68 and 749.59, supplementary data). PG6+1C18:1 was not identified with HRMS for sample S4 (Table S4, supplementary data), whereas it was found using HPLC-MS and UHPLC-MS, with a relative abundance of $1.3 \%$ to $1.4 \%$ with the two methods, for the same sample (Table S8 and S12, m/z: 749.69 and m/z 749.62, supplementary data). It appears that the development of the two chromatographic methods brings complementary resolution to HRMS for the study of these complex matrices. 
Both the HPLC and U-HPLC methods can discriminate cyclic PG, generated by ESI reaction or during the polymerisation process, which are found in various PGE structures. For sample 1, PG4+2C18 was the most abundant PGE, identified at an rt of 8.30 min with HPLC (Table S5).

401

402 An intramolecular condensation was found with the identification of PG4[1] $+2 \mathrm{C} 18$ at an rt of $9.33 \mathrm{~min}$, related to an increase in hydrophobic and steric interactions with the stationary phase. The PG3 + 1C18 monoester was identified at an rt of 1.32, and the corresponding cyclic PG3[1] $+1 \mathrm{C} 18$ was found at the same rt of 1.32 , related to ESI-induced dehydration and at $\mathrm{rt} 1.96$, also related to an increase in hydrophobic and steric interactions with the stationary phase (Table S5). The same analysis can be achieved for all samples, for a precise characterisation of these specific classes of cyclic PGEs, in accordance with the previous work of Chaimbault et al. [21] and Crowther et al. [22]. The detailed PGEs analysis with one or two water loss is identical to the analysis performed with the U-HPLC method.

\section{Conclusions}

The study of the composition of polyglycerol ester mixtures remains a challenge, even after decades of studies, as they display numerous structures that have very close features.

Thanks to the modelling of all possible PG and PGE structures up to 12 PG units and High Resolution Mass Spectrometry (HRMS), we were able to identify the compounds present in the 4 different samples provided by the Gattefossé company, and to construct a structural database with HRMS data. HPLC-MS and U-HPLC-MS analyses using a QDA detector (Waters), enabled us to perform PGE separations of PGEs in 10 minutes (HPLC) and 5 minutes (UHPLC) gradients. The detection produced extracted ion chromatograms (XICs), based on all the possible adducts identified in the HRMS analysis. Using 2 different gradient times and comparing the retention factors and the $\log \mathrm{P}$ of the different species, we were able to distinguish between different possible isobaric ions and therefore to make a reliable relative 
quantification of all the PGEs identified in order to compare different synthesised samples with each other.

\section{Acknowledgements}

The results of this study were obtained by the "Polyglycerol Ester Characterisation" research project funded by the Gattefossé company.

\section{References.}

[1] V. K. Babayan, T. G. Kaufman, H. Lehman, R. J. Tkaczuk, Some uses and applications of polyglycerol esters in cosmetic and pharmaceutical preparations. J. Soc. Cos. Chem. 15 (1964) 473-483.

[2] W. Hemker, Associative structures of polyglycerol esters in food emulsions. J. Am. Oil Chem. Soc. 58 (1981) 114-119. https://doi.org/10.1007/BF02672194

[3] K. Oppenlaender, B. Wegner, K. Stork, F. Frosch, H.U. Wekel, Use of mixtures of polyglycerol fatty esters as emulsifiers in cosmetic and pharmaceutical formulations. Patent US 5147644 (1992).

[4] N. Chailloux, V. Nardello, J.L. Salager, J.M. Aubry, Propriétés amphiphiles des tensioactifs de la famille du monolaurate de polyglycérol et application à la préparation de microémulsions à base d'esters gras. Oléagineux, Corps Gras et Lipides 10 (2003) 382-386.

\section{https://doi.org/10.1051/ocl.2003.0382}

[5] E. Mohammadifar, A. Bodaghi, A. Dadkhahtehrani, A. Nemati Kharat, M. Adeli, R. Haag, Green Synthesis of Hyperbranched Polyglycerol at Room Temperature. ACS Macro Lett. 6 (2017) 35-40. https://doi.org/10.1021/acsmacrolett.6b00804 
[6] A. B. Leoneti, V. Aragão-Leoneti, S. V. W. B. de Oliveira, Glycerol as a by-product of biodiesel production in Brazil: Alternatives for the useof unrefined glycerol. Renew. Energ. 45 (2012) 138-145. https://doi.org/10.1016/j.renene.2012.02.032

[7] R.T. Mclntyre, Polyglycerol Esters. J. Am. Oil Chem. Soc. 56 (1979) 835A-840A. https://doi.org/10.1007/BF02667458

[8] V.K. Babayan, R.T. McIntyre, Preparation and properties of some polyglycerol esters of short and medium chain length fatty acids. J. Am. Oil Chem. Soc. 48 (1971) 307-309. https://doi.org/10.1007/BF02890751

[9] J.M. Clacens, Y. Pouilloux, J. Barrault, Selective etherification of glycerol to polyglycerols over impregnated basic MCM-41 type mesoporous catalysts, Appl. Catal. A 227 (2002) 181190. https://doi.org/10.1016/S0926-860X(01)00920-6

[10] C. García-Sancho, R. Moreno-Tost, J.M. Mérida-Robles, J. Santamaría-González, A. Jiménez-López, P. Maireles Torres, Etherification of glycerol to polyglycerols over MgAl mixed oxides, Catal. Today 167 (2011) 84-90. https://doi.org/10.1016/j.cattod.2010.11.062

[11] C. Curschellas, K. Nagy, E. Windhab, H.J. Limbach, Characteristics of polyglycerol ester and its different fractions, J. Colloid Interface Sci. 393 (2013) 182-191. http://dx.doi.org/10.1016/j.jcis.2012.10.063

[12] W.R. Michael, R.H. Coots, Metabolism of polyglycerol and polyglycerol esters, Toxicol. Appl. Pharm. 20 (1971) 334-345. https://doi.org/10.1016/0041-008X(71)90277-8.

[13] H. Nosal, J. Nowicki, M. Warzała, E. Nowakowska-Bogdan, M. Zarebska, Synthesis and characterization of alkyd resins based on Camelinasativa oil and polyglycerol, Prog. Org. Coat. 86 (2015) 59-70. http://dx.doi.org/10.1016/j.porgcoat.2015.04.009

[14] T.N. Kumar, Y.S.R. Sasatry, G. Lakshminarayana, Analysis of polyglycerols by highperformance liquid chromatography, J. Chromatogr. A. 298 (1984) 360-365.

https://doi.org/10.1016/S0021-9673(01)92732-3 
[15] M. Macka, H.P. Mettler, M. Bokel, W. Röder, Analysis of silanised polyglycerols by supercritical fluid chromatography, J. Chromatogr. A. 675 (1994) 267-270. https://doi.org/10.1016/0021-9673(94)85284-7

[16] B. De Meulenaer, G.V. Royen, B. Vanhoutte, A. Huyghebaert, Combined liquid and gas chromatographic characterisation of polyglycerol fatty acid esters, J. Chromatogr. A. 896 (2000) 239-251. https://doi.org/10.1016/S0021-9673(00)00391-5

[17] S. Cassel, P. Chaimbault, C. Debaig, T. Benvegnu, S. Claude, D. Plusquellec, P. Rollin, M. Lafosse, Liquid chromatography of polyglycerol fatty esters and fatty ethers on porous graphitic carbon and octadecyl silica by using evaporative light scattering detection and mass spectrometry, J. Chromatogr. A. 919 (2001) 95-106. https://doi.org/10.1016/S00219673(01)00801-9

[18] C. Marques-Alvarez, E. Sastre, J. Perez-Pariente, Solid catalysts for the synthesis of fatty esters of glycerol, polyglycerols and sorbitol from renewable resources, Top. Catal. 27 (2004) 105-117. https://doi.org/10.1023/B:TOCA.0000013545.81809.bd

[19] C. Lafossas, F. Benoit-Marquié, J.C. Garrigues, Analysis of the retention of tetracyclines on reversed-phase columns: Chemometrics, design of experiments and quantitative structureproperty relationship (QSPR) study for interpretation and optimization, Talanta 198 (2019) 550-559. https://doi.org/10.1016/j.talanta.2019.02.051

[20] S. Fekete, R. Berky, J. Fekete, J.L. Veuthey, D. Guillarme, Evaluation of a new wide pore core-shell material (AerisTM WIDEPORE) and comparison with other existing stationary phases for the analysis of intact proteins. J. Chromatogr. A. 1236 (2012) 177-188. doi:10.1016/j.chroma.2012.03.018

[21] P. Chaimbault, S. Cassel, S. Claude, C. Debaig, T. Benvegnu, D. Plusquellec, R Rollin, M. Lafosse, Direct Analysis of Industrial Oligoglycerols by Liquid Chromatography with 
495 Evaporative Light-Scattering Detection and Mass Spectrometry, Chromatographia, 50 (1999)

496 239-242. https://doi.org/10.1007/BF02490658

497 [22] M.W. Crowther, T.R. O’Connell, S.P. Carter, Electrospray mass spectrometry for 498 characterizing polyglycerols and the effects of adduct ion and cone voltage. J. Am. Oil Chem. 499 Soc. 75 (1998) 1867-1876. https://doi.org/10.1007/s11746-998-0343-X 